\title{
MAXIMAL ELEMENTS OVER NON-COMPACT SUBSETS OF LINEAR TOPOLOGICAL SPACES
}

\author{
Nicholas C. YANNELIS * \\ University of Minnesota, Minneapolis, MN 55455, USA \\ Received 17 September 1984
}

We provide an extension of Browder's fixed point theorem which is used to prove a very general existence of maximal elements result. This result generalizes previous theorems on the existence of maximal elements. It also corrects a recent claim of Mehta.

\section{Introduction}

In a recent paper Mehta (1984) made a claim about the existence of maximal elements for binary relations defined over a non-empty subset of a Euclidean space which need not be compact or convex. Unfortunately this claim is not quite true as we will show by a simple counter-example. Fortunately the claim is true with a slight additional assumption. While we are at it, we extend his result to Hausdorff linear topological spaces. This theorem includes the previous results on maximal elements given in Fan (1962), Sonnenschein (1971), Aliprantis-Brown (1983) and Yannelis-Prabhakar (1983). Also it can be used to prove an existence of maximal elements result for weak preference relations, adopting the techniques developed in Kim-Richter (1984).

\section{Notation and definitions}

\subsection{Notation}

$2^{A}$ denotes the set of all subsets of $A$, con $A$ denotes the convex hull of the set $A$, $\backslash$ denotes the set theoretic subtraction, and $\mathbb{R}^{l} \quad$ denotes the $l$-fold Cartesian product of the set of real numbers $\mathbb{R}$.

\subsection{Definitions}

Let $X$ be a topological space. A binary relation $\mathscr{P}$ on $X$ is a subset of $X \times X$. We real $(x, y) \in \mathscr{P}$ as ' $x$ is preferred to $y$ '. Define the correspondence $P: X \rightarrow 2^{X}$ by $P(x)=\{y \in X:(y, x) \in \mathscr{P}\}$ and $P^{-1}$ : $X \rightarrow 2^{X}$ by $P^{-1}(y)=\{x \in X: y \in P(x)\}$. We call $P(x)$ the upper section or upper contour set of $P$, and

* I am grateful to Taesung Kim, Ket Richter and Bill Zame for comments, discussions and suggestions. Also I am very indebted to a competent referee for rescuing me from a mishap. 
$P^{-1}(y)$ the lower section or lower contour set of $P$. If there exists $x^{*} \in X$ such that $P\left(x^{*}\right)=\phi$, then $x^{*}$ is said to be a maximal element in $X$.

\section{Counter-example}

Mehta (1984) made the following statement:

Statement. Let $X$ be a subset of $\mathbb{R}^{\prime}$. Let $\mathscr{P}$ be a binary relation on $X$ satisfying the following conditions:

(i) for each $x \in X, x \notin P(x)$ and $P(x)$ is convex,

(ii) $P^{-1}(y)=\{x \in X: y \in P(x)\}$ is open in $X$ for each $y \in X$, and

(iii) there exists $y_{0} \in X$ such that $x \backslash P^{-1}\left(y_{0}\right)$ is compact.

Then there exists a maximal element, i.e., there exists $x^{*} \in X$ such that $P\left(x^{*}\right) \cap X=\phi$.

By means of a very simple counter-example we show that the above statement as stated is false. Let $X=\{a, b\} \subset \mathbb{R}^{1}$, with $a \neq b$. Obviously, $X$ is not convex and it has the discrete topology. Define the correspondence $P: X \rightarrow 2^{X}$ by $P(a)=\{b\}$ and $P(b)=\{a\}$. Note that $P$ satisfies all the conditions of the statement; but there is no maximal element. Therefore, the statement is false. However, we show in Theorem 2 below how the statement becomes true with a slight additional assumption.

\section{A fixed point theorem}

In this section we provide a fixed point theorem which slightly generalizes Browder's (1968) fixed point result. We will first need the following extension of the Knaster-Kuratowski-Mazurkiewicz (K-K-M) lemma proved in Fan (1962).

Lemma. Let $X$ be an arbitrary set in a Hausdorff linear topological space $Y$. For each $x \in X$ let $F(x)$ be a closed set in $Y$ satisfying

(i) The convex hull of any finite subset $\left\{x_{1}, \ldots, x_{n}\right\}$ of $X$ is contained in $\bigcup_{i=1}^{n} F\left(x_{i}\right)$, and

(ii) $F(x)$ is compact for at least one $x \in X$.

Then $\bigcap_{x \in X} F(x) \neq \phi$.

Theorem 1. Let $X$ be a non-empty, convex, closed subset of a Hausdorff linear topological space $Y$ and $\phi: X \rightarrow 2^{X}$ be a correspondence such that :

(i) $\phi(x)$ is convex and non-empty for all $x \in X$,

(ii) for each $y \in X$ the set $\phi^{-1}(y)=\{x \in X: y \in \phi(x)\}$ is open in $X$, and

(iii) there exists $y_{0} \in X$ such that $X \backslash \phi^{-1}\left(y_{0}\right)$, is compact.

Then there exists $x^{*} \in X$ such that $x^{*} \in \phi\left(x^{*}\right)$.

Proof. Suppose otherwise, i.e., for all $x \in X, x \notin \phi(x)$. Let for each $y \in X, F(y)=X \backslash \phi^{-1}(y)$. Since for each $y \in X, \phi^{-1}(y)$ is open in $X, F(y)$ is closed in $X$ for each $y \in X$, and it is obviously closed in 
$Y$ as well, since $X$ is a closed subset of $Y$. It follows from assumption (iii) that $F(y)$ is compact for some $y \in X$. In order to apply the $\mathrm{K}-\mathrm{K}-\mathrm{M}$ lemma we need to show that the convex hull of any arbitrary collection of points $\left\{y_{1}, \ldots, y_{n}\right\}$ from $X$ is contained in $\bigcup_{i=1}^{n} F\left(y_{i}\right)$. Suppose not, then there exists $x \in \operatorname{con}\left\{y_{1}, \ldots, y_{n}\right\}$ and $x \notin \bigcup_{i=1}^{n} F\left(y_{i}\right)$. Then for all $i=1, \ldots, n, x \in \phi^{-1}\left(y_{i}\right)$ or $y_{i} \in \phi(x)$ for all $i$ and therefore $x \in \operatorname{con}\left\{y_{1}, \ldots, y_{n}\right\} \subset \operatorname{con} \phi(x)=\phi(x)$, a contradiction to $x \notin \phi(x)$ for all $x \in X$. Hence, by the $\mathrm{K}-\mathrm{K}-\mathrm{M}$ lemma we have that $\bigcap_{y \in X} F(y) \neq \phi$. Let $z \in \bigcap_{y \in X} F(y)$. Then for all $y \in X$, $z \notin \phi^{-1}(y)$ which implies that $\phi(z)=\phi$, some $z \subset Z$. But this contradicts our assumption that $\phi$ is non-empty valued. Hence, there exists $x^{*} \in X$ such that $x^{*} \in \phi\left(x^{*}\right)$. Q.E.D.

Note that the proof of the above theorem is different from the one given in Browder (1968) [see also Yannelis-Prabhakar (1983, Theorem 3.3)], which is based on an argument using partitions of unity. In fact, the above proof seems to be simpler. It is clear that, if in Theorem $1 X$ is assumed to be compact, then one obtains as a corollary Browder's fixed point theorem.

\section{An existence of maximal elements theorem}

We now give our existence of maximal elements results, extending Mehta's ideas. It is proved by means of the fixed point theorem given in the previous section.

Theorem 2. Let $X$ be a convex, non-empty, closed subset of a Hausdorff linear topological space and $P: X \rightarrow 2^{X}$ be a preference correspondence such that:

(i) $x \notin \operatorname{con} P(x)$ for all $x \in X$,

(ii) for all $y \in X, P^{-1}(y)=\{x \in X: y \in P(x)\}$ is open in $X$, and

(iii) there exists $y_{0} \in X$ such that $X \backslash\left\{x \in X: y_{0} \in \operatorname{con} P(x)\right\}$ is compact.

Then there exists $x^{*} \in X$ such that $P\left(x^{*}\right)=\phi$.

Proof. Suppose otherwise, i.e. for all $x \in X, P(x) \neq \phi$. Define the correspondence $\phi: X \rightarrow 2^{X}$ by $\phi(x)=\operatorname{con} P(x)$ for all $x \in X$. By Lemma 5.1 in Yannelis-Prabhakar (1983) $\phi$ has open lower sections, i.e., the set $\phi^{-1}(y)=\{x \in X: y \in \phi(x)\}$ is open in $X$ for each $y \in X$. Furthermore, $\phi$ is non-empty, convex valued, and from assumption (iii) it follows that $X \backslash \phi^{-1}(y)$ is compact for some $y \in X$. Hence, by Theorem 1 there exists $x^{*} \in X$ such that $x^{*} \in \phi\left(x^{*}\right)=\operatorname{con} P\left(x^{*}\right)$, a contradiction to assumption (i). Q.E.D.

Remark 1. Theorem 2 is a slight generalization of Theorem 5.1 in Yannelis-Prabhakar (1983). It also includes the results on the existence of maximal elements of Aliprantis-Brown (1983), Fan (1962) and Sonnenschein (1971).

Remark 2. Note that since $X \backslash\{x \in X: y \in \operatorname{con} P(x)\} \subset X \backslash P^{-1}(y)$ the assumption (iii) in Theorem 2 is weaker than the assumption in the statement that: there exists $y_{0} \in X$ such that $X \backslash P^{-1}\left(y_{0}\right)$ is compact.

Remark 3. The result of Mehta (1984) becomes true if $X \geqq \mathbb{R}^{l}$ is convex and closed, and this is a straight forward corollary of Theorem 2.

Remark 4. Recently, Kim-Richter (1984) have obtained very general existence of maximal elements results for weak preference relations. Using their techniques it can be shown that a similar result with Theorem 2 holds for weak preference relations. 


\section{References}

Aliprantis, C. and D. Brown, 1983, Equilibria in markets with a Riesz space of commodities, Journal of Mathematical Economics 11, 189-207.

Borglin, A. and H. Keiding, 1976, Existence of equilibrium actions and of equilibrium: A note on the 'new' existence theorems, Journal of Mathematical Economics 3, 313-316.

Browder, F., 1968, The fixed-point theory of multivalued mappings in topological vector spaces, Mathematische Annalen 177 , 283-301.

Fan, K., 1962, A generalization of Tychonoff's fixed point theorem, Mathematische Annalen 142, 305-310.

Kim, T. and M.K. Richter, 1984, Non-transitive consumer theory, Mimeo.

Mehta, G., 1984, Maximal elements for non-transitive binary relations, Economics Letters 14, 163-165.

Sonnenschein, H., 1971, Demand theory without transitive preferences with applications to the theory of competitive equilibrium, in: J. Chipman et al., eds., Preferences, utility and demand (Harcourt, Brace, Jovanovich, New York).

Yannelis, N.C. and N.D. Prabhakar, 1983, Existence of maximal elements and equilibria in linear topological spaces, Journal of Mathematical Economics 12, 233-245. 